\title{
COMPARISON OF TWO INTRAOSSEOUS ACCESS DEVICES EMPLOYED DURING SIMULATED CARDIOPULMONARY RESUSCITATION. A PROSPECTIVE, RANDOMIZED, CROSSOVER, MANIKIN STUDY
}

\author{
Jorge Garau Ramirez ${ }^{1}$, Zenon Truszewski ${ }^{2}$, Anna Drozd ${ }^{3}$ \\ ${ }^{1}$ University of Valencia, Valencia, Spain \\ ${ }^{2}$ Department of Emergency Medicine, Medical University of Warsaw, Poland \\ ${ }^{3}$ International Institute of Rescue Research and Education, Poland
}

\begin{abstract}
BACKGROUND: Intraosseous injection is an alternative method used regarding unsuccessful intravenous access during many emergency situations. The aim of the present study was to compare injections made by the Bone Injection Gun (BIG) with NIO Adult intraosseous access devices during simulated CPR performed by paramedics.

METHODS: 40 paramedics took part in this prospective, randomized, crossover, manikin study. The participants were chosen at random, while each paramedic performed an intraosseous injection with the Bone Injection Gun (BIG) or with the NIO Adult Intraosseous access device. The effectiveness of the intraosseous injection was analyzed as times $\mathrm{T1}, \mathrm{T} 2$, and $\mathrm{T} 3$. Time $\mathrm{T} 1$ is defined as the time-lapse from placing the intraosseous device into one's hand to performing the intraosseous injection; Time $\mathrm{T} 2$ is the time-lapse from placing the intraosseous device into one's hand to the moment of stabilizing it at the injection site; while Time T3 is defined as the time-lapse from putting the intraosseous device into one's hand, attaching the syringe with a test aspiration, to connecting the infusion line. Attitudes toward the use of intraosseous access during resuscitation were also analyzed in the present study.

RESULTS: The efficacy of intraosseous access obtained with the use of $\mathrm{NIO}$ was at $100 \%$ where the efficacy of the use of BIG was at $95 \%$. The average time of $\mathrm{T} 1$ was similar in the groups randomized to use BIG and NIO, represented as $5.4 \pm 3.5$ vs. $3.5 \pm 2.5$ s, respectively $(p=0.014)$; the average time of T2 was $17.5 \pm 4.5 \mathrm{vs} .3 .5 \pm 2.5 \mathrm{~s}$, respectively $(p<0.001)$; while the average time of T3 was $25 \pm 5.5$ vs. $11.5 \pm 2.5 \mathrm{~s}$, respectively $(p<0.001)$. Notably, $90 \%$ of the study's participating paramedics preferred to use the NIO during cardiopulmonary resuscitation $(p<0.001)$.
\end{abstract}

CONCLUSIONS: The present study shows that after a short period of training paramedics can perform an intraosseous injection with a high degree of efficiency. Thus, the authors stress the need for training medical personnel to have the skill to perform intraosseous injections along with knowledge and understanding of the indications and contraindication for 10 access.

KEY WORDS: intraosseous access, paramedic, cardiopulmonary resuscitation, simulation, education. 


\section{INTRODUCTION}

One of the basic skills that should be encompassed by medical personnel in the field of emergency medicine is performing an intravascular injection. An injection as such not only provides access to medication but provides access to fluid intake in order to expand the vascular bed and increase blood pressure $[1,2]$. In emergency medicine, many cases occur requiring intravascular access that should be obtained as soon as possible [3-6]. Such emergency cases may include the onset of anaphylactic and hypovolemic shock and the necessity to initiate CPR. The importance of intravenous access during CPR is marked by the presence of a nonshockable rhythm described as pulseless electrical activity (PEA) or asystole during which the key step is the administration of adrenalin as outlined by the guidelines of the European Resuscitation Council (ERC) [7, 8], and the American Heart Association (AHA) $[9,10]$. Intravascular access, however, may prove difficult as in the case of sudden cardiac arrest (SCA) in which there is a collapse of the vascular bed with which peripheral intravenous access (most commonly near the cuboidal fossa) may be not only difficult, but impossible. Moreover, while in departments of Emergency Medicine, Anaesthesiology and Intensive Care Units a trained anaesthesiologist may be able to obtain central line access, this is an impossible undertaking for paramedics. In their training, paramedics lack the qualified technique training, as well as access to the necessary instruments. Interosseous access (IO), therefore, serves as an alternative approach in the pre-hospital and hospital setting. This interosseous access may be obtained by devices such as the puncture Bone Injection Gun (BIG) and the NIO Adult Intraosseous device, both of which are compared in the present study.

Both intraosseous access devices provide intraosseous access with a semi-automatic technique in which by pressing the release trigger, the needle is ejected. This form of needle insertion proves useful in obtaining access into the intraosseous cavity. With this in mind, the aim of the current study was to compare the two types of devices under simulated CPR performed by paramedics.

\section{METHODS}

The present study was approved by the Institutional Review Board of the International Institute of Rescue Research and Education (approval no:

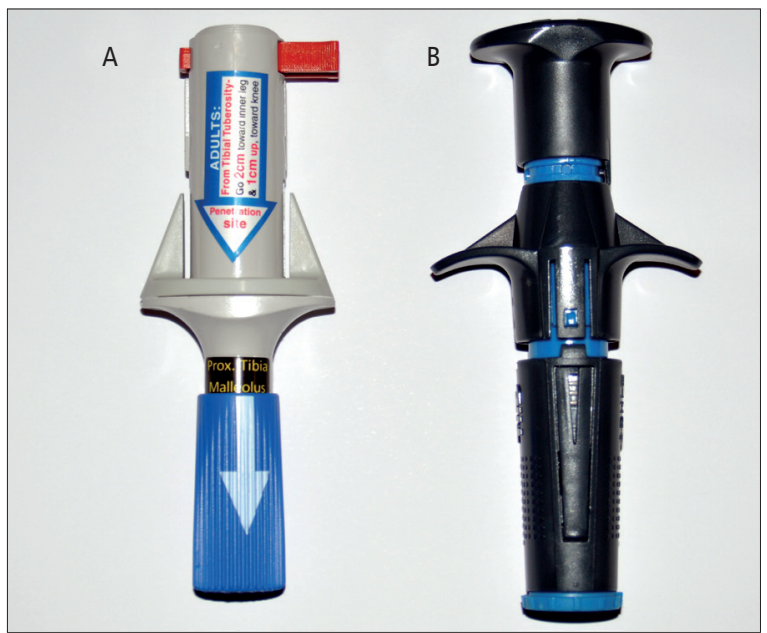

FIGURE 1. Intraosseous access devices used in the study: (A) Bone Injection Gun; (B) NIO Adult Intraosseous device

23.05.2016.01). After the presentation of the study's objectives, 44 paramedics participated in the study.

All of the participants before commencing the study completed a questionnaire concerning their knowledge and skill regarding intraosseous injection. Subsequently, all participants received appropriate training regarding the indications, contraindications, and techniques used in order to gain intraosseous access.

Once the theoretical training concluded, an instructor demonstrated the correct technique of performing intraosseous injection with both 10 devices, namely; the Bone Injection Gun (BIG, WaisMed Ltd., Rosh Ha'Ayin, Israel) and the NIO Adult device (NIO; Persys Medical, Houston, TX, USA) (Fig. 1).

Participants in a randomized crossover order performed the intraosseous injection under conditions of simulated CPR. The ResearchRandomizer program (www.randomizer.org) was used to determine the order of participant participation, as well as which IO access devices each would use for the first then the second trial. The detailed randomized procedure is shown in Figure 2. At first, the first group performed intraosseous injections using the BIG intraosseous device while the other used the $\mathrm{NIO}$ device. Following a 30 minute break, the participants performed injections with the other $1 \mathrm{O}$ devices. The intraosseous injections were performed using the Stat Adult ALS Manikin with the intraosseous Leg Trainer (Simulaids, Saugerties, NY, USA).

The intraosseous access success was defined as an attempt to introduce the intraosseous injection into the correct location, which proved to be a suc- 


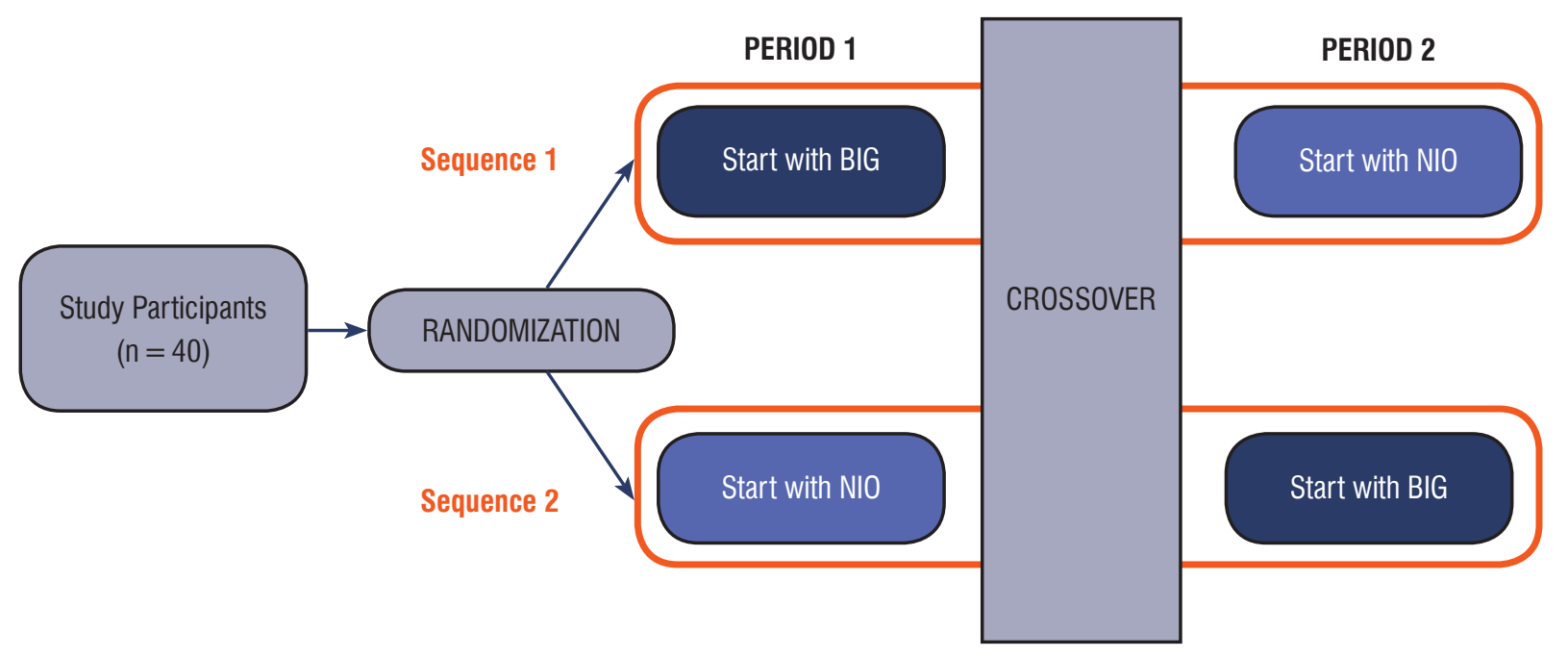

FIGURE 2. Randomization flow chart

cessful outcome. Furthermore, the study measured three time parameters as measures of the effectiveness of the intraosseous injection. Time T1 was the time lapse from placing the intraosseous device into one's hand and performing the intraosseous injection with the needle; Time T2 was measured as the time lapse from placing the intraosseous device into one's hand to the moment of stabilizing it at the injection site; while Time T3 was the time lapse from placing the intraosseous device into one's hand, attaching the syringe with a test aspiration, to connecting the infusion line. The participants were also asked to indicate the ease of intraosseous access using the given equipment and specify which 10 device they would most likely use in real CPR conditions.

All the data collected was evaluated using the Statistica Package Software, version 12.5. Results were given as absolute values, percentages, medians, interquartile ranges (IQR) or means, and SD. To check for normal distribution, the Kolmogorov-Smirnov test was used. As a randomized crossover trial study, pairings were taken into consideration in the statistical analysis. McNemar's test was used to compare the cannulation success rates of the humeral head and the proximal tibia whereas the two-sided Wilcoxon signed-rank test allowed one to compare the procedure time. The participants' subjective opinions were compared with the use of the Stuart-Maxwell test. The value of $p>0.05$ was considered statistically significant.

\section{RESULTS}

In the present study, 40 paramedics (12 females, equivalent to $30 \%$ of the participants) with an ave- rage age of $26.5 \pm 3.7$ years and work experience of $4.3 \pm 2.5$ years took part. Only 5 of the 40 participants $(12.5 \%)$ stated that they had had previous experience with $1 \mathrm{O}$ access. Amongst these, the average number of completed 10 access devices uses was $3 \pm 1$. None of the study participants had previous experience with the use of the NIO Adult Intraosseous device.

To answer at which point during the resuscitation the participants believed an intraosseous injection would prove beneficial; $60 \%$ of the participants indicated that they would attempt $1 \mathrm{O}$ access after two minutes of unsuccessful attempts at IV access, whereas, $40 \%$ of participants would attempt 10 access after a single unsuccessful attempt at IV access. Furthermore, the participants described the contraindications to intraosseous injection as follows: fracture of limbs (100\%); infection of the tissue at the site of planned injection (85\%); extremity trauma with damage to the vascular bundle nerves (80\%); and compartment syndrome (32.5\%). In addition, $7.5 \%$ of the participants indicated that 10 access needed to be obtained within the first 24 hours. The participants also reported the complications of $\mathrm{IO}$ access, namely: the dissection of bone (75\%); bleeding (72.5\%); osteomyelitis (52.5\%); and infection at the injection site (10\%). Moreover, $42.5 \%$ of participants indicated that medication administered via 10 achieves the desired plasma concentration in a time which is comparable to those given via central line access, while $30 \%$ believed that the time compares to access obtained via the peripheral vein. Furthermore, $27.5 \%$ of participants indicated that the desired plasma level concentration is achieved at a longer time interval 
when administered via 10 access as compared to peripheral access.

The present study found that the efficacy for 10 access using NIO was at $100 \%$ and $95 \%$ for the use of the BIG device. The lower efficacy with the use of BIG may be attributed to the too small setting of the depth of the puncture. All of the participants were able to correctly identify the place of 10 access at the proximal tibia.

The average $\mathrm{T} 1$ time in the group using $\mathrm{BIG}$ and NIO device was comparable and amounted to $5.4 \pm 3.5$ vs. $3.5 \pm 2.5$ s, respectively $(p=0.014)$; the average T2 amounted to $17.5 \pm 4.5$ vs. $3.5 \pm 2.5 \mathrm{~s}$, respectively $(p<0.001)$; while the average T3 time was $25 \pm 5.5$ vs. $11.5 \pm 5.2$ s, respectively $(p<0.001)$. Although not a statistically significant difference $(p=0.534)$, the participants did disclose that NIO was a simpler way to perform the intraosseous injection as compared to BIG. However, 90\% of the study's participating paramedics, preferred to use the NIO during cardiopulmonary resuscitation $(p<0.001)$.

\section{DISCUSSION}

This study is, to our knowledge, the first that compares the efficacy of BIG and NIO intraosseous access devices during simulated cardiopulmonary resuscitation performed by paramedics.

Although in the standards of paediatric care intraosseous access is the technique of choice for intravascular access, in adults it is used in the event of failed vascular access, as in the case of an emergency scenario in which CPR is required. This is also true regarding the need to establish intravascular access in trauma patients (without arrest). In emergency situations, intraosseous access is becoming the recommended method with failed or prolonged attempts at intravenous access. Furthermore, the Advanced Trauma Life Support guidelines [11] outline that intraosseous access should be obtained after three unsuccessful attempts at access to the peripheral vein, or after 2 minutes of attempts.

Intraosseous injection provides entry for resuscitation drugs and fluid resuscitation [12-14]. However, flow through the 10 catheter must be initiated by an initial flush of at least $10 \mathrm{~mL}$ of saline and maintained with the use of a pressure infusion bag inflated at $300 \mathrm{mmHg}$ in accordance with the instructions of use [15]. Notably, the medication given via an intraosseous injection achieves the desired plasma concentration which compares to that by a central line catheter [16].

Undoubtedly, central vein cannulation is the superior method as compared with intraosseous access. However, as shown in the research, the effectiveness of access, the duration of the procedure, and the possible complications, suggest 10 access to be the most accessible method. Several studies comparing the complications of 10 access have reported complications such as iatrogenic bone fracture, osteomyelitis, and tissue necrosis [17-20]. Alternative studies, nonetheless, have reported no complications found as a result of 10 access [21]. The research also explains that there are varying locations of 10 access such as the tibia, the head of humerus [22-26] or the sternum [27] which may be used for IO access. In cardiopulmonary resuscitation, however, the research has demonstrated that the effectiveness of obtaining 10 access to the proximal tibia (Fig. 3) is more likely than that to the humeral head $[21,23,28]$. This is especially significant regarding the present study, as it is, to the knowledge of its researchers, the first one to compare the efficacy of $\mathrm{BIG}$ and NIO intraosseous access devices employed during a simulated cardiopulmonary resuscitation performed by paramedics.

The results of this study further demonstrate that 10 access is a rapid and simple way to obtain intravascular access in emergency scenarios. However, the present study does illustrate certain limitations as its data was collected under simulated conditions in order to avoid possible complications which could arise with human subjects. Although the study's participant pool only included paramedics, which may be presented as an another limitation, this was

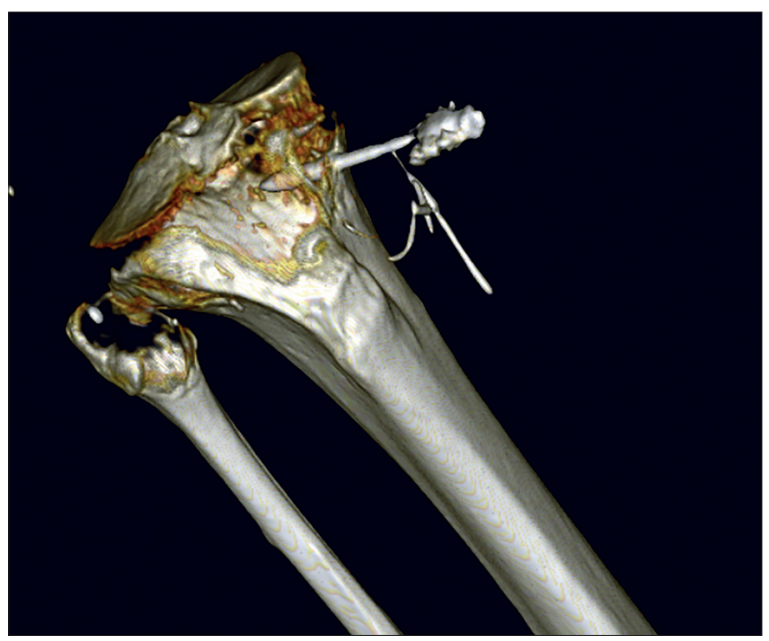

FIGURE 3. Intraosseous needle view in CT scan 
deliberate considering it is paramedics which are most often in need of employing 10 access in CPR emergency scenarios.

Even with such limitations present, it is also important to note that in order to mimic chest compression the study employed the Lifeline ARM (ARM, Defibtech, Guilford, CT, USA) which the research shows to be sufficient as compared with manual chest compressions $[29,30]$. The use of the Lifeline ARM, as a result, caused a lower degree of error as unequal manual chest compression was avoided. The study's design, as one which was a randomized, crossover study, the researchers also believe to be an advantage.

\section{CONCLUSIONS}

The present study shows that after a short period of training paramedics can perform intraosseous injections with a high degree of efficiency. Thus, the authors stress the need for training medical personnel to have the skill to perform intraosseous injections along with knowledge and understanding of the indications and contraindication for 10 access.

\section{Conflict of interest statement}

The authors declare that they have no conflict of interest regarding any financial or personal relationship with the manufacturers or with any other people or organizations that could inappropriately influence or bias their work.

\section{Role of funding source}

The authors declare that they received no funding or any other kind of sponsorship regarding study design, data collection, and analysis as well as submitting the manuscript for publication. This study was only funded by Department of Emergency Medicine, Medical University of Warsaw funding.

\section{REFERENCES}

1. Wolfson DL, Tandoh MA, Jindal M et al. Adult Intraosseous Access by Advanced EMTs: A Statewide Non-Inferiority Study. Prehosp Emerg Care, 2016; 5: 1-7.

2. Smereka J, Madziała M, Szarpak L. Are firefighters able to perform intraosseous access and start fluid resuscitation in an anaphylactic patient? Am J Emerg Med, 2016; 34(8): 1707-1708. doi: 10.1016/ j.ajem.2016.05.068.

3. Chreiman KM, Kim PK, Garbovsky LA, Schweickert WD. Blueprint for Implementing New Processes in Acute Care: Rescuing Adult Patients with Intraosseous Access. J Trauma Nurs, 2015; 22(5): 266-273. doi: 10.1097/JTN.0000000000000152.

4. Benson G. Intraosseous access to the circulatory system: An under-appreciated option for rapid access. J Perioper Pract, 2015; 25(7-8): 140-143.

5. Szarpak L, Truszewski Z, Fudalej M, Krajewski P. The intraosseous access devices as a method of vascular access during cardiopulmonary resuscitation. Am J Emerg Med, 2016; 34(2): 321-322. doi: 10.1016/ j.ajem.2015.11.013.

6. Szarpak L, Truszewski Z, Smereka J et al. Ability of paramedics to perform intraosseous access. A randomized cadaver study comparing EZ-IO(®) and NIO(®) devices. Resuscitation, 2016; 104: e5-e6. doi: 10.1016/j.resuscitation.2016.04.011.

7. Soar J, Nolan JP, Böttiger BW et al. European Resuscitation Council Guidelines for Resuscitation 2015: Section 3. Adult advanced life support. Resuscitation, 2015; 95: 100-147. doi: 10.1016/j.resuscitation.2015.07.016.

8. Maconochie IK, Bingham R, Eich C et al. European Resuscitation Council Guidelines for Resuscitation 2015: Section 6. Paediatric life support. Resuscitation, 2015; 95: 223-248. doi: 10.1016/j.resuscitation.2015.07.028.

9. de Caen AR, Berg MD, Chameides L et al. Part 12: Pediatric Advanced Life Support: 2015 American Heart Association Guidelines Update for Cardiopulmonary Resuscitation and Emergency Cardiovascular Care. Circulation, 2015; 132(18 Suppl 2): S526-542. doi: 10.1161/ CIR.0000000000000266.

10. Link MS, Berkow LC, Kudenchuk PJ et al. Part 7: Adult Advanced Cardiovascular Life Support: 2015 American Heart Association Guidelines Update for Cardiopulmonary Resuscitation and Emergency Cardiovascular Care. Circulation, 2015; 132 (18 Suppl 2): S444-464. doi: 10.1161/CIR.0000000000000261.

11. Bouillon B, Probst C, Maegele $M$ et al. Emergency room management of multiple trauma: ATLS ${ }^{\circ}$ and $S 3$ guidelines. Chirurg, 2013; 84(9): 745-752. doi: 10.1007/s00104-013-2476-1.

12. Szarpak $Ł$, Czyzewski $Ł$, Woloszczuk-Gebicka B et al. Comparison of $\mathrm{NIO}$ and EZ-IO intraosseous access devices in adult patients under resuscitation performed by paramedics: a randomized crossover manikin trial. Am J Emerg Med, 2016; 34(6): 1166-1167. doi: 10.1016/ j.ajem.2016.03.017.

13. Hammer N, Möbius R, Gries A et al. Comparison of the Fluid Resuscitation Rate with and without External Pressure Using Two Intraosseous Infusion Systems for Adult Emergencies, the CITRIN (Comparison of InTRaosseous infusion systems in emergency medicINe)-Study. PLoS One, 2015; 10(12): e0143726. doi: 10.1371/journal.pone.0143726.

14. Ker K, Tansley G, Beecher D et al. Comparison of routes for achieving parenteral access with a focus on the management of patients with Ebola virus disease. Cochrane Database Syst Rev, 2015; 26(2): CD011386. doi: 10.1002/14651858.CD011386.pub2.

15. Pasley J, Miller CH, DuBose JJ et al. Intraosseous infusion rates under high pressure: a cadaveric comparison of anatomic sites. J Trauma Acute Care Surg, 2015; 78(2): 295-299. doi: 10.1097/ TA.0000000000000516. 
16. Wenzel V, Lindner KH, Augenstein S et al. Intraosseous vasopressin improves coronary perfusion pressure rapidly during cardiopulmonary resuscitation in pigs. Crit Care Med, 1999; 27(8): 1565-1569.

17. Hasan MY, Kissoon N, Khan TM et al. Intraosseous infusion and pulmonary fat embolism. Pediatr Crit Care Med, 2001; 2(2): 133-138.

18. Bowley DM, Loveland J, Pitcher GJ. Tibial fracture as a complication of intraosseous infusion during pediatric resuscitation. J Trauma, 2003; 55(4): 786-787.

19. Stoll E, Golej J, Burda G. Osteomyelitis at the injection site of adrenalin through an intraosseous needle in a 3-month-old infant. Resuscitation, 2002; 53(3): 315-318.

20. Henson NL, Payan JM, Terk MR. Tibial subacute osteomyelitis with intraosseous abscess: an unusual complication of intraosseous infusion. Skeletal Radiol, 2011; 40(2): 239-242. doi: 10.1007/ s00256-010-1027-9.

21. Paxton JH, Knuth TE, Klausner HA. Proximal humerus intraosseous infusion: a preferred emergency venous access. J Trauma, 2009; 67(3): 606-611. doi: 10.1097/TA.0b013e3181b16f42.

22. Levitan RM, Bortle CD, Snyder TA et al. Use of a battery-operated needle driver for intraosseous access by novice users: skill acquisition with cadavers. Ann Emerg Med, 2009; 54(5): 692-694. doi: 10.1016/ j.annemergmed.2009.06.012.

23. Szarpak L, Truszewski Z, Smereka J et al. A Randomized Cadaver Study Comparing First-Attempt Success Between Tibial and Humeral Intraosseous Insertions Using NIO Device by Paramedics: A Preliminary Investigation. Medicine (Baltimore), 2016; 95(20): e3724. doi: 10.1097/MD.0000000000003724.
24. Kurowski A, Timler D, Evrin T, Szarpak L. Comparison of 3 different intraosseous access devices for adult during resuscitation. Randomized crossover manikin study. Am J Emerg Med, 2014; 32(12): 1490-1493. doi: 10.1016/j.ajem.2014.09.007.

25. Zasko P, Szarpak L, Kurowski A et al. Success of intraosseous access procedure in simulated adult resuscitation. Crit Care Resusc, 2016; 18(2): 134.

26. Szarpak Ł, Truszewski Z, Smereka J. Which intravascular access method to choose during cardiopulmonary resuscitation? Am J Emerg Med, 2016; 34(6): 1160. doi: 10.1016/j.ajem.2016.02.061.

27. Findlay J, Johnson DL, Macnab AJ et al. Paramedic evaluation of adult Intraosseous Infusion System. Prehosp Disaster Med, 2006; 21(5): 329-334.

28. Warren DW, Kissoon N, Sommerauer JF, Rieder MJ. Comparison of fluid infusion rates among peripheral intravenous and humerus, femur, malleolus, and tibial intraosseous sites in normovolemic and hypovolemic piglets. Ann Emerg Med, 1993; 22(2): 183-186.

29. Szarpak $Ł$, Truszewski Z, Smereka J, Czyżewski $Ł$. Does the use of a chest compression system in children improve the effectiveness of chest compressions? A randomized crossover simulation pilot study. Kardiol Pol, 2016 Jul 8. doi: 10.5603/KP.a2016.0107.

30. Kurowski A, Szarpak Ł, Bogdański Ł et al. Comparison of the effectiveness of cardiopulmonary resuscitation with standard manual chest compressions and the use of TrueCPR and PocketCPR feedback devices. Kardiol Pol, 2015; 73(10): 924-930. doi: 10.5603/KP.a2015.0084. 